\title{
Intraoral Sensory Abnormalities in A Patient with Juvenile Fibromyalgia
}

\author{
Takuya Naganawa*1, Abhishek Kumar ${ }^{2}$, Hitoshi Sato ${ }^{1}$, Toshihiro Okamoto ${ }^{1}$ and Tomohiro Ando ${ }^{1}$ \\ ${ }^{1}$ Department of Oral and Maxillofacial Surgery, Tokyo Women's Medical University, Japan
}

${ }^{2}$ Department of Dental Medicine, Karolinska Institutet, Sweden

Submission: January 30, 2018; Published: August 24, 2018

*Corresponding author: Takuya Naganawa, Department of Oral and Maxillofacial Surgery, Tokyo Women's Medical University School of Medicine, 8-1 Kawada-cho, Shinjuku-ku, Tokyo 162-8666, Japan; Email: tanaganawa@gmail.com

Abstract

A 9-year-old Japanese girl was referred to our institution with symptoms of intraoral burning pain. The patient had been diagnosed with juvenile fibromyalgia 6 month earlier. Intraoral qualitative sensory testing showed mechanical allodynia and heat hyperalgesia on the intraoral mucosa (tongue and cheek). Because of the lack of evidence for peripheral, organic changes in the oral mucosa, the sensory changes may be best explained by peripheral disturbance. According to the above findings, we diagnosed the patient with burning mouth syndrome (neuropathy), and we consider an association may exist between these reported somatosensory changes and JFM. The psychological managements by a child psychiatry specialist and hospitalization for changing their environments were performed. Moreover, the tooth brushing technique with avoiding the sites of allodynia was instructed carefully. After the 6 months of the management, the body pain and intraoral burning pain were reduced 7 to 2 on a NRS. The continuous dental managements may be important because of the difficulties of dental treatments in a condition of intraoral burning pain with allodynia [1-5].

Keywords: Juvenile fibromyalgia, Allodynia, Sensory changes, Intraoral qualitative sensory testing; Peripheral; Allodynia; Parts of body; Neuropathic pain; Brushing technique; Dental treatments; Patients; Tongue; Cheek; Similar Drugs; Hyperacusis; Knowledge

Abbreviations: JFM: Juvenile Fibro Myalgia; QST: Quantitative Sensory Testing; ACR: American College of Rheumatology

\section{Introduction}

Juvenile fibromyalgia (JFM) is a non-inflammatory chronic pain condition that most frequently affects the children at approximately 9-15 years of age. The male: female occurrence ratio of JFM is about 1: 4-8. It is a common, multifactorial disease, diagnosed on the basis of the total sum of painful illness, chronic fatigue, hypothermia, and many other autonomic signs / symptoms. JFM is characterized by constant widespread pain in different parts of body, poor sleep quality, daytime sleepiness and an altered mood. Various clinical forms of JFM are reported previously, however, to the best of our knowledge, there is no information about intraoral manifestations and somatosensory changes (neuropathy etc.) associated with JFM. Here, we report the case of a patient with JFM who exhibited intraoral somatosensory abnormalities [6].

\section{Case report}

A 9-year-old Japanese girl was referred to the Department of Oral and Maxillofacial Surgery, Tokyo Women's Medical University Hospital, with symptoms of intraoral burning pain 7 on a numerical rating scale (NRS) ranging $0-10$ ). The patient was previously diagnosed with JFM 6 month earlier. The patient was wearing sun glasses at all the time for protection of dazzling and reported hyperacusis. The patient reported difficulty in tooth brushing due to intraoral burning pain although intraoral examination revealed fair oral health status. An Intraoral Quantitative Sensory Testing (QST) with a Q-tip was performed to investigate mechanical allodynia and sensitivity to warm and cold perceptions were tested with a thermal device [7] The QST tests performed on the intraoral mucosa indicated mechanical allodynia on the tongue and cheeks, bilaterally. Although the NRS of heat stimuli on the tongue and cheek were high, there was no differences between right and left sides. According to the above findings, we diagnosed the patient with burning mouth syndrome (neuropathic pain).

The psychological managements by child psychiatry specialist and hospitalization for changing their environments were performed. Moreover, the results of intraoral sensory testing were identified with the patient, and then the tooth brushing technique with avoiding the sites of allodynia was instructed carefully. After the 6 months of the management, the body pain and intraoral burning pain were reduced 7 to 2 on 


\section{Open Access Journal of Neurology \& Neurosurgery}

a NRS. The continuous dental managements may be important because of the difficulties of dental treatments in a condition of intraoral burning pain with allodynia [7].

\section{Discussion}

JFM is characterized by constant widespread pain in different parts of the body, poor sleep quality, daytime sleepiness and an altered mood. The quality of life of individuals with chronic pain and their caregivers is severely restricted and the occurrence of symptoms of anxiety and depression is fairly common in this population. In the present case the patient wore protective eyeshades and reported hyperacusis. Thus, the medical interview and the sensory testing were performed quiet room by an examiner (TN). An empirical observation revealed that the patient shared a cordial relation with her mother contrary to the personality trait developed by youngest patients (ref). However, the patient seems to be stressed by her academic exams [8-11].

Patients complain of pain of unknown etiology in the musculoskeletal system (myalgia and arthralgia) as well as persistent headache. Intraoral somatosensory changes are not well described because of difficulties in sensory testing in the intraoral region due to the specific structure of the oral cavity. In present case, we used intraoral thermal devices specifically developed for intraoral sensory testing, which we previously demonstrated had sufficient reliability [7]. We identified mechanical allodynia and heat hyperalgesia at intraoral mucosa (tongue and cheek). The intraoral thermal devices are small in size, light weight and easy access to intraoral cavity. Although our previous study on the reliability measurements of the thermal device was for adult participants, the intraoral thermal device may be possible to use for the sensory testing in the children. To our knowledge, no previous reports have described the abnormalities of intraoral sensory loss or gain in JFM patients. Because of the lack of evidence for peripheral, organic changes in the oral mucosa, sensory changes may be best explained by peripheral disturbance. We therefore consider an association may exist between these reported somatosensory changes and JFM.

Non-steroidal anti-inflammatory drugs are used, although less effectively, for the treatment of pain associated with juvenile fibromyalgia. Neurotropin $®$ is often used as a specific therapeutic agent but its efficacy has not been validated. Various drug therapies have been tried based on experience with adult cases: selective serotonin re-uptake inhibitor, as well as selective serotonin/noradrenalin re-uptake inhibitor, have been reported to be effective. Gabapentin (Gabapen $\AA$ ), which was developed as an anti-epileptic agent, has also proved effective for neurogenic pain and is used in this disease. In contrast to adult cases, however, it is difficult to use antidepressants or gabapentin, or similar drugs, for JFM because of off-label age and adverse events. Moreover, these agents are not proved to be efficacious in a trial with a small number of children. Pregabalin, tramadol, and mirtazapine have recently been developed for adults and these agents should be systematically tested if they are to be clinically used.

In this case, the psychological management with hospitalization was performed. Consultation (three times a week) by child psychiatry specialist and the temporarily isolation from her home environment were effective in this case. Following the results of the intraoral sensory testing, the diagnosed 'burning mouth syndrome (neuropathic pain) associated with JFM' were identified. The identified symptoms were shared with the patient, and then the tooth brushing technique was instructed carefully with avoiding the painful sites. The pain was relieved 7 to 2 on a NRS without any medication treatments. Juvenile fibromyalgia has not been sufficiently appreciated thus far. Case collection is so sparse that specific diagnostic criteria have not been created. The most frequently used diagnostic criteria are those for adults that were set forth by the American College of Rheumatology (ACR) in 1990. These criteria include the following as central findings: pains persist for $3>=$ month over a wide area of the body and tender points are detected at 18 site. The tender point can be detected only when an appropriate site is pressed and it evades detection if a pressure site is distant from the appropriate site by as little as $5 \mathrm{~mm}$. Physical examination of the joints as well as of the muscles of the entire body is crucial, including simultaneous detection of tender points, which is indispensable for making a diagnosis of juvenile fibromyalgia. The intraoral finding was not including these diagnostic criteria, moreover, there are no information of intraoral manifestation and sensory changes so far. Somatosensory changes were also found to be associated with the present case of JFM. The mechanisms of peripheral neurological changes of JFM have not yet been clarified, however, intraoral somatosensory changes related to JFM may be due to progressive lesions associated with this disease. Thus, continuous follow-up and sensory testing to assess disease progression may be helpful to clarify these issues. To our knowledge, this report is the first to describe intraoral manifestations and somatosensory changes in a patient with JFM. Therefore, a greater accumulation of cases is needed to clarify intraoral abnormalities of JFM patients.

\section{References}

1. Naganawa T, Baad-Hansen L, Iida T, Ando T, Svensson P (2015) Assessment of human intraoral thermal sensitivity with simple devices in the clinic: implications for orofacial pain conditions. J Oral Facial Pain Headache 29(1): 83-90.

2. Yokota S, Kikuchi M, Miyamae T (2013) Juvenile fibrimyalgia: Guidance for management. Pediatr Int 55(4): 403-409.

3. Wolfe F, Smythe HA, Yunus MB, Bennett RM, Bombardier C, et al (1990) The American College of Rheumatology 1990 criteria for the classification of fibromyalgia: Report of the multicenter criteria committee. Arthritis Rheum 33(2):160-172.

4. Yunus MB, Masi AT (1985) Juvenile primary fibromyalgia syndrome. A clinical study of thirty-three patients and matched normal controls. Arthritis Rheum 28(2): 138-1345.

5. Kashikar-Zuck S, Graham TB, Huenefeld MD, Power SW (2000) Juvenile primary fibromyalgia syndrome. Arthritis Care Res 13: 388-397. 
6. Yokota S (2009) Juvenile fibromualgia: Guidance for diagnosis and management. In: Nishioka K (Ed.) Guideline for Management of Fibromyalgia. Medical Review, Tokyo, pp.75-82.

7. Yokota S, Umebayashi H, Miyamae T (2007) Our experience of 3 cases with childhood fibromyalgia. J Jpn Pediatr Soc 111: 462-468.

8. Miyamae T, Yokota S (2008) The actual state and clinical features of fibromyalgia in our country. J Jpn Pediatr Soc 112: 1769-1777.

9. Naganawa T, Sato H, Kumar A, Iida T, Naganawa E, Okamoto T Ando $\mathrm{T}$ (2015) Clinical Presentation of Oral Manifestations and Intraoral Somatosensory Changes in Fahr's Disease. J Pain Relief 4: 214-216.
10. Naganawa T, Kumar A, Sato H, Uchida J, Okamoto T Ando T. Intraoral sensory abnormalities caused by tooth extraction in a patient with chronic inflammatory demyelinating polyneuropathy. J Clin Neurosci In Submission.

11. Naganawa T, Baad-Hansen L, Ando T, Svensson P (2015) Influence of topical application of capsaicin, menthol and local anesthetics on intraoral somatosensory sensitivity in healthy subjects-temporal and spatial aspects. Exp brain Res 233(4): 1189-1199.

\begin{tabular}{|l|}
\hline \multicolumn{1}{|c|}{ Your next submission with Juniper Publishers } \\
will reach you the below assets \\
- Quality Editorial service \\
- Swift Peer Review \\
- Reprints availability \\
- E-prints Service \\
- Manuscript Podcast for convenient understanding \\
- Global attainment for your research \\
- Manuscript accessibility in different formats \\
( Pdf, E-pub, Full Text, Audio) \\
- Unceasing customer service \\
Track the below URL for one-step submission \\
https://juniperpublishers.com/online-submission.php
\end{tabular}

